\title{
Expression pattern and level of ING5 protein in normal and cancer tissues
}

\author{
XUE-FENG YANG ${ }^{1}$, DAO-FU SHEN ${ }^{1}$, SHUANG ZHAO $^{1}$, TIAN-REN REN ${ }^{2}$, YANG GAO $^{1}$, \\ SHUAI SHI ${ }^{1}$, JI-CHENG WU ${ }^{1}$, HONG-ZHI SUN ${ }^{1}$ and HUA-CHUAN ZHENG ${ }^{1,3}$ \\ ${ }^{1}$ Cancer Center and Key Laboratory of Brain and Spinal Cord Injury of Liaoning Province, \\ The First Affiliated Hospital of Jinzhou Medical University, Jinzhou, Liaoning $121001 ;{ }^{2}$ Jilin Province Forestry Bureau, \\ Linjiang, Jilin 134600; ${ }^{3}$ Institute of Life Sciences, Jinzhou Medical University, Jinzhou, Liaoning 121001, P.R. China
}

Received February 21, 2016; Accepted February 13, 2017

DOI: $10.3892 / \mathrm{ol} .2018 .9581$

\begin{abstract}
Inhibitor of growth family 5 (ING5) functions as a type-II tumor suppressor gene and exerts an important role in DNA repair, apoptotic induction, proliferative inhibition, chromatin remodeling and the invasion process. In the present study, immunohistochemistry was performed to characterize the expression profile of ING5 protein on a tissue microarray containing mouse and human normal tissues, and human cancer tissues, including hepatocellular $(n=62)$, renal clear cell $(n=62)$, pancreatic $(n=62)$, esophageal squamous cell $(n=45)$, cervical squamous cell $(n=31)$, breast $(n=144)$, gastric $(n=196)$, colorectal $(n=96)$, endometrial $(n=96)$ and lung carcinoma $(n=192)$. In the mouse tissues, ING5 expression was detected in the cytoplasm of neurons, the nephric tubule and glomerulus, alveolar epithelium, gastrointestinal glands, squamous epithelium of the skin and skeletal muscles. By contrast, ING5 was localized to the cell nucleus in breast tissues. In human tissues, ING5 protein was primarily localized in the cytoplasm. However, ING5 was detected in the cytoplasm and nucleus in various types of normal tissues, including the tongue, stomach, intestine, lung and breast. In total, ING5 expression was detected in 400/986 cancer tissues (40.6\%). In the majority of cases, ING5 expression was observed to be restricted to the cytoplasm. However, ING5 was also detected in the nucleus in a number of cancer tissues, including gastric, colorectal and lung carcinoma. Notably, ING5 was more frequently expressed in breast $(79.9 \%)$, colorectal $(56.3 \%)$ and endometrial carcinoma (50.0\%). The incidence of ING5 expression in hepatocellular carcinoma (14.5\%) and pancreatic carcinoma (22.6\%) was low. These findings indicate that ING5
\end{abstract}

Correspondence to: Professor Hua-Chuan Zheng, Cancer Center and Key Laboratory of Brain and Spinal Cord Injury of Liaoning Province, The First Affiliated Hospital of Jinzhou Medical University, 5-2 Renmin Street, Jinzhou, Liaoning 121001, P.R. China E-mail: zheng_huachuan@hotmail.com

Key words: inhibitor of growth family 5, expression profile, immunohistochemistry, mouse, human, cancer may be involved in cell regeneration and be associated with colorectal carcinogenesis.

\section{Introduction}

Inhibitor of growth family 5 (ING5) is a member of the ING protein family and functions as a type-II tumor suppressor gene. Human ING5 is mapped to chromosome 2 q37.3 and encodes a $28-\mathrm{kDa}$ protein with 240 amino acids (1). As indicated in Fig. 1, ING5 protein consists of a number of domains, including leucine zipper like (LZL), novel conserved region (NCR), nuclear localization signal (NLS) and plant homeodomain (PHD). Among them, the LZL domain is important in DNA repair, apoptotic induction and chromatin remodeling, whereas the NCR domain can bind to histone acetyl transferase (HAT) complexes during chromatin remodeling and the regulation of gene expression $(2,3)$. ING5 interacts with histone $\mathrm{H} 3 \mathrm{~K} 4 \mathrm{me} 3$ and is involved in the formation of HAT complexes (ING5-histone acetyltransferase KAT7-JADE and ING5-monocytic leukemia zinc finger protein-MOZ-related factor-bromodomain-PHD finger protein $(1,2)$.

ING5 protein is able to bind to mini-chromosome maintenance proteins, which is important for DNA replication via the formation of a pre-replicative complex (3). ING5 was reported to activate the cyclin-dependent kinase inhibitor p21/cyclin dependent kinase inhibitor $1 \mathrm{~A}$ promoter to induce its expression, promote the acetylation of p53 at Lys-382 residues and interact with the p300 protein of HAT complexes (2-6). ING5 is a cofactor of Tip60 for the acetylation of p53 at K120 in response to DNA damage (7). Previously, reduced ING5 expression was detected in pancreatic carcinoma cells transfected with a microRNA (miR)-196a precursor, accompanied by decreased apoptosis, increased invasion and proliferation compared with control cells (8). Additionally, low-level laser irradiation treatment was able to induce the pro-proliferation effects of miR-193 on bone mesenchymal stem cells, which is mediated via an ING5 inhibitor (9).

In the RKO colorectal cancer cell line, ING5 overexpression results in decreased colony-forming efficiency, decreased cell population in the $\mathrm{S}$ phase and $\mathrm{p} 53$-dependent apoptosis induction $(10,11)$. In a previous study, the loss of heterozygosity on the long arm of chromosome 2, where the ING5 gene is 
located, was detected in 85\% (33/39) of oral carcinoma cases. Reduced ING5 mRNA expression in $61 \%$ of oral squamous cell carcinoma cases with missense mutations located within the LZL finger and NCR domains of the ING5 protein has also been reported (12). In head and neck squamous cell carcinoma (HNSCC), nuclear ING5 may modulate the transactivation of target genes, and promote apoptosis and cell cycle arrest by interacting with p300 and p21 $(13,14)$. Additionally, two truncated fragments of ING5 (aa 1-184 and aa 107-226) are able to induce cellular senescence via the downregulated expression of cyclin E and cyclin dependent kinase 2 (13). Reduced nuclear expression and cytoplasmic translocation of ING5 was observed in HNSCC, gastric and colorectal carcinoma tumorigenesis (14-16).

Identification of tissues or cell types which express ING5 will contribute towards the elucidation of its physiological function. Additionally, the clarification of the expression pattern of ING5 and heterogeneity between tumor cases will contribute to the development of target gene therapy and conditional animal knockout models of ING5. In the present study, an intermittent microwave irradiation for immunohistochemistry of ING5 was employed, where the microwave irradiation causes minute vibrations ( $>2.4$ billion times/sec) and increases the probability of specific antibody-antigen reactions (17). The expression profiling of ING5 protein has been investigated in normal mouse and human tissues, as well as in human cancer tissues.

\section{Materials and methods}

Amino acid sequence alignment. Amino acid sequences of human and mouse ING5 were obtained from GenBank (18), including their isoforms. These sequences were aligned using Genetyx 7 from Genetyx Corporation (Tokyo, Japan).

Tissue specimens and tissue microarray. Written informed consent was obtained for the use of tumor tissues $(n=986)$ for clinical research, and ethical approval was obtained from the Ethical and Animal Experimentation committees at Jinzhou Medical University (Jinzhou, China).

C57BL/6 mice (3 males and 3 females; 8 weeks old) were purchased from Beijing HFK Bioscience Co., Ltd. (Beijing, China) and housed in pathogen-free conditions in a temperature-controlled animal room with a 12-h light/dark illumination cycle. All had ad libitum access to standard rodent food and water. They were sacrificed under sodium pentobarbital anesthesia, and the resected samples included brain, heart, liver, spleen, lung, kidney, breast, stomach and intestine. All tissues were fixed in $10 \%$ neutral formalin, embedded in paraffin and cut into $4 \mathrm{~mm}$ sections. The tissue arrays of human normal tissues (cerebrum, cerebellum, brain stem, aorta, tongue, thyroid, esophagus, stomach, intestine, liver, pancreas, lung, trachea, appendix, smooth muscle, skeletal muscle, heart, testis, bladder and prostate) and cancer tissues [hepatocellular carcinoma $(n=62)$, renal clear cell carcinoma $(n=62)$, pancreatic carcinoma $(n=62)$, esophageal squamous cell carcinoma $(n=45)$ and cervical squamous cell carcinoma $(n=31)]$ were purchased from Shanghai Outdo Biotech Co., Ltd (Shanghai, China). The human cervix, endometrium, ovary and breast tissues were obtained from surgical samples in The First
Affiliated Hospital of Jinzhou Medical University (Jinzhou, China). Breast $(n=144)$, gastric $(n=196)$, colorectal $(n=96)$, endometrial $(n=96)$ and lung carcinoma $(n=192)$ tissues were also collected from The First Affiliated Hospital of Jinzhou Medical University. Dissected and collected specimens from The First Affiliated Hospital of Jinzhou Medical University were subjected to routine preparation of formalin (10\%)-fixed (room temperature for $48 \mathrm{~h}$ ) and paraffine-embedded tissue block, and the performance of tissue microarray using tissue microarrayer (KIN-1; Azumaya Co., Ltd., Warabi, Japan). None of the patients with cancer underwent chemotherapy, radiotherapy or adjuvant treatment prior to surgical resection between January 1, 2008 and Dececember 30, 2015.

Immunohistochemistry. Consecutive sections were dewaxed with xylene, rehydrated with graded alcohol (100, 90, 80, 70 and $60 \%$ ) to water and subjected to antigen retrieval in a bioled target retrieval solution (Dako; Agilent Technologies, Inc., Santa Clara, CA, USA) in a microwave oven (Panasonic Corporation, Osaka, Japan) for $15 \mathrm{~min}$ at $100^{\circ} \mathrm{C}$. Sections were subsequently blocked in $5 \%$ bovine serum albumin (Sigma-Aldrich; Merck KGaA, Darmstadt, Germany) for 5 min to prevent non-specific antibody binding. The sections were incubated with a rabbit anti-ING5 antibody (dilution, 1:50; cat. no. 11560-1-AP; ProteinTech Group, Inc., Chicago, IL, USA) for $15 \mathrm{~min}$ at $37^{\circ} \mathrm{C}$, followed by incubation with an anti-rabbit secondary antibody conjugated to horseradish peroxidase (1:100; cat no. P0399; Dako; Agilent Technologies, Inc.) for $15 \mathrm{~min}$ at $37^{\circ} \mathrm{C}$. All of the incubations were performed at $37^{\circ} \mathrm{C}$ for $15 \mathrm{~min}$ in a microwave oven as previously described (3). Following each treatment, the slides were washed three times (1 min each) with TBST. Bound antibodies were visualized using 3,3'-diaminobenzidine. The sections were counterstained with Mayer's hematoxylin, dehydrated, cleared and mounted. Normal rabbit immunoglobulin G (1:100; cat no. 500-P00; PeproTech China, Suzhou, China) was used instead of the primary antibody as a negative control.

Immunostaining. ING5 protein was observed under a light microscope (BX43; Olympus Corporation, Tokyo, Japan; x200). Initially, a strong expression field was selected under low magnification, and 100 cells were randomly counted from five distinct and representative fields of each section blindly by 2 independent pathologists. The percentage of counted cells was scored as follows: Negative, $0-10 \%$ and positive, $11-100 \%$.

Statistical analysis. A $\chi^{2}$ test was performed to compare the positive rates of different groups. SPSS 10.0 software (SPSS, Inc., Chicago, IL, USA) was used for analysis. $\mathrm{P}<0.05$ was considered to indicate a statistically significant difference.

\section{Results}

As presented in Fig. 2, the subcellular location of ING5 was cytoplasmic or nucleo-cytoplasmic in the mouse tissues with either a sporadic or local distribution. There was variation in ING5 expression among the different tissues. ING5 reactivity was detectable in the cytoplasm of neurons, the nephric tubule and glomerulus, alveolar epithelium, glands of the stomach and intestine, squamous epithelium of the skin and skeletal 


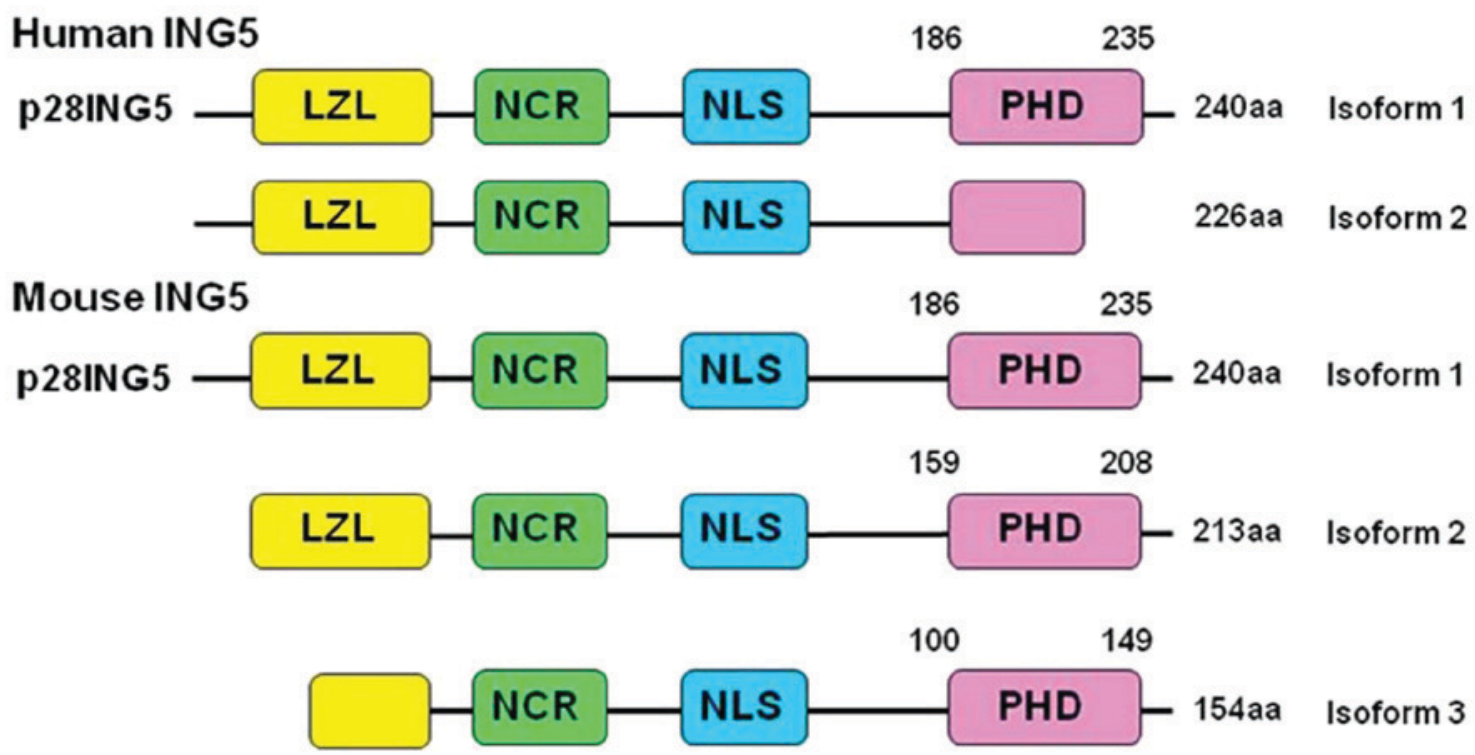

Figure 1. Schematic diagram of the human and mouse isoforms of ING5. aa, amino acid; ING5, inhibitor of growth family 5; LZL, leucine zipper like; NCR, novel conserved region; NLS, nuclear localization signal; PHD, plant homeodomain.

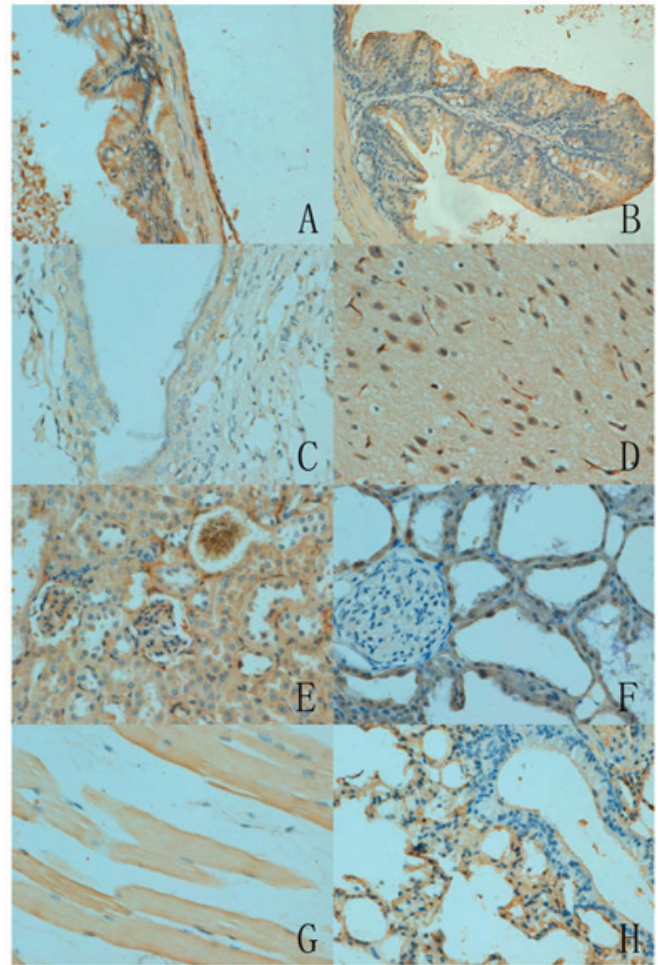

Figure 2. ING5 protein expression in normal mouse tissues (magnification, x200). (A) Stomach, (B) intestine, (C) kidney, (D) brain, (E) kidney, (F) breast, $(\mathrm{G})$ heart, $(\mathrm{H})$ lungs and bronchial tissues. ING5, inhibitor of growth family 5 .

muscles. Furthermore, ING5 was also detected in the nucleus of the breast glandular epithelium (Table I).

In human tissues, the ING5 protein was principally distributed in the cytoplasm. ING5 expression was detected in the cytoplasm and nucleus in a number of tissue types, including the tongue, stomach, intestine, lung and breast (Fig. 3). According to the density, strong immunoreactivity of ING5 was detected in the tongue, stomach and skin, whereas weak immunoreactivity was observed in the cerebellum, brain stem, thymus and skeletal muscle (Table II).

In total, ING5 expression was detected in 400/986 cancer tissues $(40.6 \%)$, with a homogenous expression pattern (Fig. 4; Table III). In the majority of cases, ING5 expression was restricted to the cytoplasm of cancer cells. ING5 expression was also detected in the nucleus and cytoplasm of gastric, colorectal and lung cancer. Notably, ING5 was more frequently expressed in breast $(79.9 \%, 115 / 144)$, colorectal $(56.3 \%, 54 / 96)$ and endometrial cancer $(50.0 \%, 48 / 96)$ compared with hepatocellular $(14.5 \%, 9 / 62)$ and pancreatic cancer $(22.6 \%, 14 / 62$, $\mathrm{P}<0.05)$.

\section{Discussion}

ING5 is a member of the ING family and is involved in DNA repair, apoptotic induction, chromatin remodeling, cellular senescence and proliferative suppression (1-3). There is an NLS in the C-terminal of ING5, which mediates its nuclear import (19). In the present study, the expression profile and cellular localization of the ING5 protein were characterized in normal mouse and human tissues, as well as in human cancer tissues. ING5 expression was primarily detected in the cytoplasm of normal mouse and human tissues, and human cancer tissues. Additionally, ING5 expression was detected in the cytoplasm and nuclei of gastrointestinal glands, the squamous epithelium of the skin, tongue and breast tissue. These results indicate that the expression pattern of ING5 has cell-specific features, and ING5 has distinct functions in different types of cells.

The phosphorylation of ING1 by 14-3-3 family (20) or Src (21) is able to induce the cytoplasmic relocalization of ING1 for apoptotic induction. The degradation of ING3 by a cytoplasmic Skp1-cullin-F-box protein complex-mediated ubiquitin-proteasome system provides further evidence for the cytosolic localization of ING3 protein (22). Therefore, 
Table I. Immunohistochemical analysis of ING5 in normal mouse tissues.

\begin{tabular}{llc}
\hline $\begin{array}{l}\text { Organ } \\
\text { or tissue }\end{array}$ & \multicolumn{1}{c}{ Cell types } & $\begin{array}{c}\text { Expression } \\
\text { pattern }\end{array}$ \\
\hline Brain & Neuron & - \\
Heart & Sporadic & + \\
Lung & Alveolar epithelium & - \\
Kidney & Nephric tubule and glomerulus & - \\
Stomach & Glandular epithelium & - \\
Intestine & Glandular epithelium & - \\
Spleen & Sporadic & + \\
Skin & Squamous epithelium & - \\
Muscle & Striated muscle cell & - \\
Liver & Sporadic & + \\
Breast & Glandular epithelium & - \\
\hline
\end{tabular}

+ , presence; -, absence. ING5, inhibitor of growth family 5 .

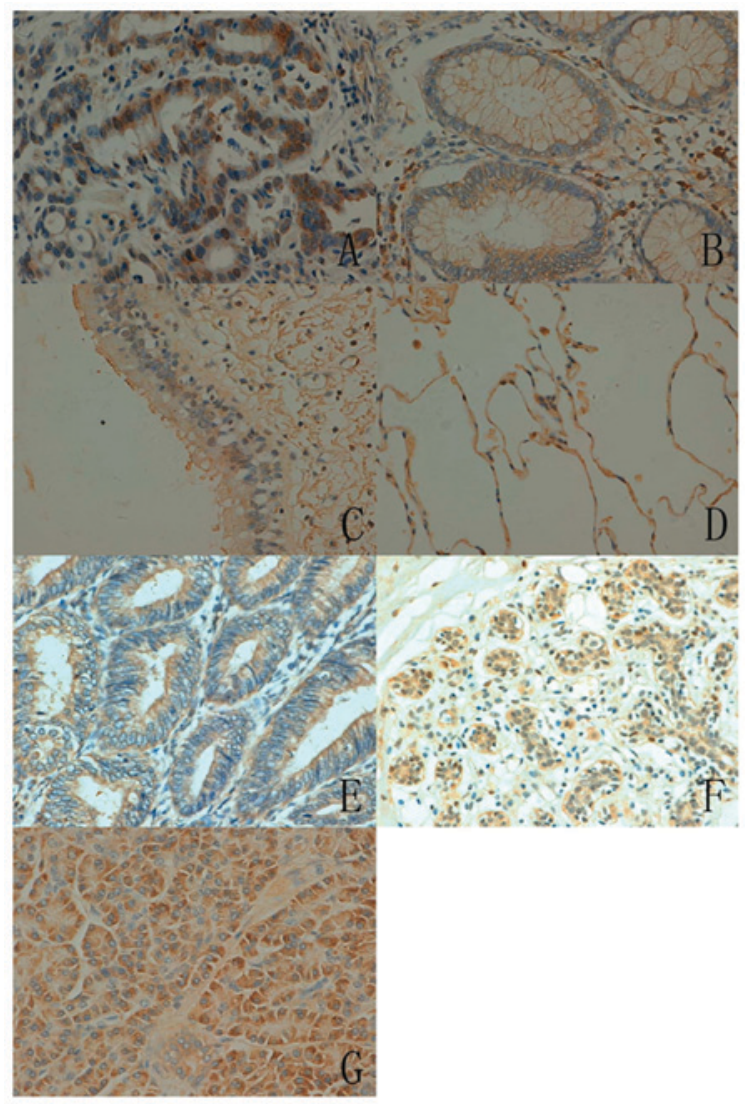

Figure 3. Immunostaining of the ING5 protein in normal human tissues (magnification, x200). (A) Stomach, (B) intestine, (C) bronchus, (D) lungs, (E) endometrium, $(\mathrm{F})$ breast and (G) pancreatic tissues. ING5, inhibitor of growth family 5 .

the authors of the present study hypothesize that the chemical modification of ING5 causes its relocalization to the cytoplasm.

Amino acid sequence alignment indicated a high similarity between human and mouse ING5 as they share $>90 \%$
Table II. ING5 protein expression in normal human tissues.

\begin{tabular}{|c|c|c|}
\hline \multirow[b]{2}{*}{ Tissue type } & \multicolumn{2}{|c|}{ ING5 expression } \\
\hline & Nucleus & Cytoplasm \\
\hline Cerebrum & - & + \\
\hline Cerebellum & - & + \\
\hline Brain stem & - & + \\
\hline Thymus & - & + \\
\hline Heart muscle & - & + \\
\hline Aorta & - & + \\
\hline Tongue & + & + \\
\hline Thyroid & - & + \\
\hline Esophagus & - & + \\
\hline Stomach & + & + \\
\hline Intestine & + & + \\
\hline Liver & - & + \\
\hline Pancreas & - & + \\
\hline Lung & + & + \\
\hline Trachea & - & + \\
\hline Skin & - & + \\
\hline Appendix & - & + \\
\hline Smooth muscle & - & + \\
\hline Skeletal muscle & - & + \\
\hline Heart & - & + \\
\hline Testis & - & + \\
\hline Bladder & - & + \\
\hline Prostate & - & + \\
\hline Cervix & - & + \\
\hline Endometrium & - & + \\
\hline Ovary & - & + \\
\hline Breast & + & + \\
\hline
\end{tabular}

+ , presence; -, absence. ING5, inhibitor of growth family 5 .

identity of the amino acid sequence (Fig. 1) (18). The present study identified no marked differences in the patterns of ING5 expression between mouse and human non-cancerous tissue samples. In human normal tissues, strong expression of ING5 was detected in in the tongue, stomach and skin. By contrast, weak expression was detected in the human cerebellum, brain stem, thymus and skeletal muscle tissues. These findings suggest the functional involvement of ING5 in specific cell types. Therefore, a conditional knockdown of ING5 will be performed using a cell-specific cre mouse in order to establish an ING5 knockout mouse model of cancer in the future.

ING5 protein has been reported to be able to inhibit cell growth, induce apoptosis and remodel chromatin by interacting with p53 and EP300/p300 (1-3). Therefore, ING5 overexpression was detectable in the tongue, skin and breast, which display a higher cell regeneration, compared with muscle and nerve tissue. ING5 is a candidate tumor suppressor gene, and its expression is downregulated in numerous types of tumors $(14,15)$. In the present study, the investigation focused on epithelial tumors and 
Table III. ING5 protein expression in human cancer tissues.

\begin{tabular}{|c|c|c|c|c|c|}
\hline \multirow[b]{2}{*}{ Type of carcinoma } & \multirow[b]{2}{*}{ Total cases $(n)$} & \multirow[b]{2}{*}{ Positive cases (n) } & \multirow[b]{2}{*}{ Positive rate $(\%)$} & \multicolumn{2}{|c|}{ ING5 expression } \\
\hline & & & & Nucleus & Cytoplasm \\
\hline Hepatocellular & 62 & 9 & 14.5 & - & + \\
\hline Renal clear cell & 62 & 20 & 32.3 & - & + \\
\hline Pancreatic & 62 & 14 & 22.6 & - & + \\
\hline Esophageal & 45 & 14 & 31.1 & - & + \\
\hline Cervical & 31 & 13 & 41.9 & - & + \\
\hline Breast & 144 & 115 & 79.9 & - & + \\
\hline Gastric & 196 & 63 & 32.1 & + & + \\
\hline Colorectal & 96 & 54 & 56.3 & + & + \\
\hline Endometrial & 96 & 48 & 50.0 & - & + \\
\hline Lung & 192 & 50 & 26.0 & + & + \\
\hline
\end{tabular}

+ , presence; -, absence. ING5, inhibitor of growth family 5 .

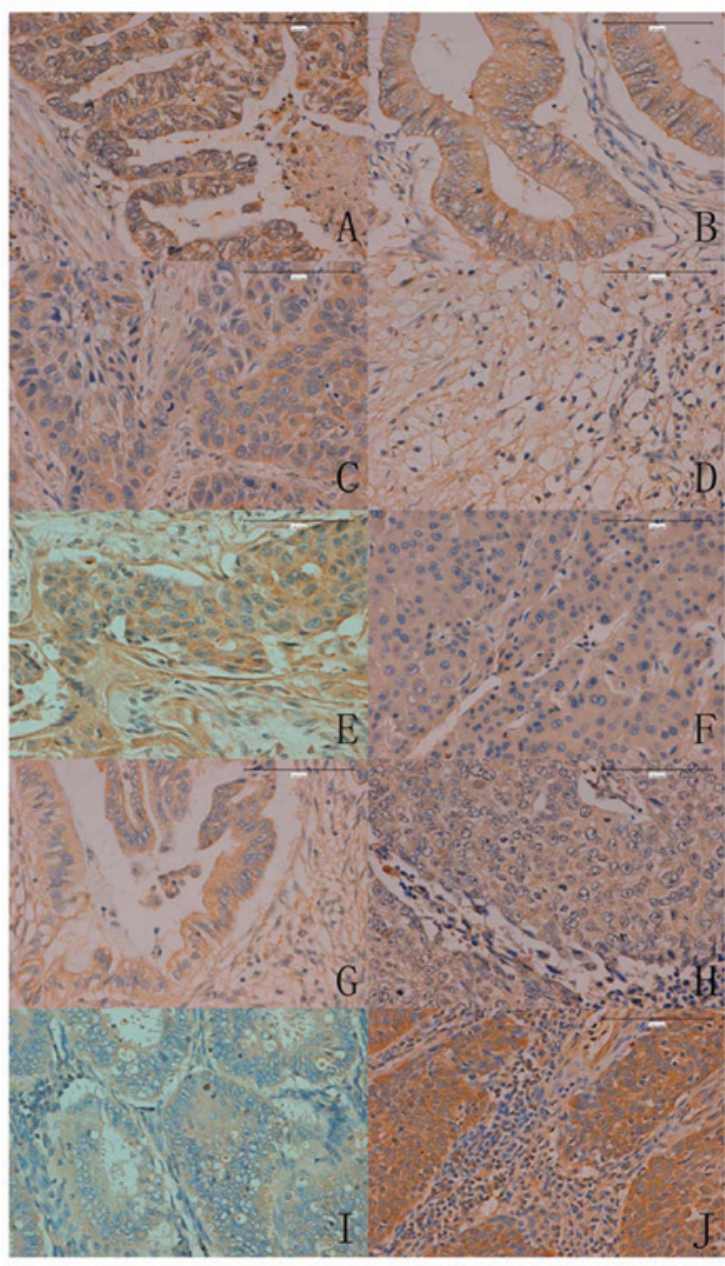

Figure 4. ING5 expression in human cancer issues (magnification, x200) (A) Gastric, (B) colorectal, (C) esophageal, (D) renal, (E) breast, (F) hepatocellular, (G) pancreatic, (H) lung, (I) endometrial and (J) cervical carcinoma. ING5, inhibitor of growth family 5 .

demonstrated that there is a high incidence of ING5 expression in gynecological cancer types, including endometrial and cervical cancer. This indicates that ING5 protein may be involved in estrogen production or regulated by estrogen. Notably, the highest level of cytoplasmic ING5 expression was detected in colorectal cancers, suggesting that cytoplasmic IN5 expression may be closely associated with colorectal carcinogenesis.

In a previous study conducted by the authors, nucleo-cytoplasmic translocation of ING5 occurred during colorectal carcinogenesis. Additionally, cytoplasmic ING5 expression was positively correlated with depth of invasion, lymphatic invasion, and TNM staging of colorectal cancer, while the inverse was true for nuclear ING5 expression (15). In the present study, the positive rate of ING5 protein was 56.3\% in colorectal cancer, but hepatocellular and pancreatic cancer exhibited lower ING5 expression at a lower positive rate $(<25 \%)$. This would significantly facilitate the identification of cancer patients who may potentially benefit from an ING5-targeted therapy. Together with these findings, the profiling of ING5 expression can be helpful to clarify the role of ING5 in proliferation and apoptosis of various types of epithelial cancer. In the present study, ING5 protein was detected in the cytoplasm of cancer cells, which was contrary to the results of other previous reports (14-16). This discrepancy in the findings may be attributed to the use of distinct sampling and fixation methods.

In summary, the present study examined ING5 expression patterns in normal mouse and human tissues, as well as in human cancer tissues. The differential expression and/or subcellular location of ING5 in various types of tissues and cells were also analyzed. ING5 expression may affect cell regeneration and be closely associated with colorectal carcinogenesis.

\section{Acknowledgements}

Not applicable.

\section{Funding}

The present study was supported by the Liaoning BaiQianWan Talents Program, Outstanding Scientific Fund 
of Shengjing Hospital, Award for Liaoning Distinguished Professors, Shenyang Science and Technology Grand (grant no. 18-013-0-59) and the National Natural Scientific Foundation of China (grant no. 81472544 and 81672700).

\section{Availability of data and materials}

The datasets used and/or analyzed during the present study are available from the corresponding author on reasonable request.

\section{Authors' contributions}

XFY, DFS, SZ, TRR, YG, SS and JCW conducted the experiments and analyzed the data. HCZ and HCS designed the study and finished the organization and writing.

\section{Ethics approval and consent to participate}

The human sample collection and animal protocols were approved by the Ethics Committee of our hospital of Jinzhou Medical University. Writing consent was provided by all patients for the present research.

\section{Patient consent for publication}

The patients consented to publication.

\section{Competing interests}

The authors declare that they have no competing interests.

\section{References}

1. Gunduz M, Gunduz E, Rivera RS and Nagatsuka H: The inhibitor of growth (ING) gene family: Potential role in cancer therapy. Curr Cancer Drug Targets 8: 275-284, 2008.

2. Soliman MA and Riabowol K: After a decade of study-ING, a PHD for a versatile family of proteins. Trends Biochem Sci 32: 509-519, 2007.

3. Wang Y, Wang J and Li G: Leucine zipper-like domain is required for tumor suppressor ING2-mediated nucleotide excision repair and apoptosis. FEBS Lett 580: 3787-3793, 2006.

4. Unoki M, Kumamoto K, Takenoshita S and Harris CC: Reviewing the current classification of inhibitor of growth family proteins Cancer Sci 100: 1173-1179, 2009.

5. Champagne KS, Saksouk N, Peña PV, Johnson K, Ullah M, Yang XJ, Côté J and Kutateladze TG: The crystal structure of the ING5 PHD finger in complex with an H3K4me3 histone peptide. Proteins 72: 1371-1376, 2008.

6. Ullah M, Pelletier N, Xiao L, Zhao SP, Wang K, Degerny C, Tahmasebi S, Cayrou C, Doyon Y, Goh SL, et al: Molecular architecture of quartet MOZ/MORF histone acetyltransferase complexes. Mol Cell Biol 28: 6828-6843, 2008.

7. Liu M, Du Y, Gao J, Liu J, Kong X, Gong Y, Li Z, Wu H and Chen H: Aberrant expression miR-196a is associated with abnormal apoptosis, invasion and proliferation of pancreatic cancer cells. Pancreas 42: 1169-1181, 2013.
8. Liu N, Wang J, Wang J, Wang R, Liu Z, Yu Y and Lu H: ING5 is a Tip60 cofactor that acetylates p53 in response to DNA damage. Cancer Res 73: 3749-3760, 2013.

9. Wang J, Huang W, Wu Y, Hou J, Nie Y, Gu H, Li J, Hu S and Zhang H: MicroRNA-193 pro-proliferation effects for bone mesenchymal stem cells after low-level laser irradiation treatment through inhibitor of growth family, member 5. Stem Cells Dev 21: 2508-2519, 2012

10. Shiseki M, Nagashima M, Pedeux RM, Kitahama-Shiseki M, Miura K, Okamura S, Onogi H, Higashimoto Y, Appella E, Yokota J and Harris CC: p29ING4 and p28ING5 bind to p53 and p300 and enhance p53 activity. Cancer Res 63: 2373-2378, 2003.

11. Cengiz B, Gunduz M, Nagatsuka H, Beder L, Gunduz E, Tamamura R, Mahmut N, Fukushima K, Ali MA, Naomoto Y, et al: Fine deletion mapping of chromosome 2q21-37 shows three preferentially deleted regions in oral cancer. Oral Oncol 43: 241-247, 2007.

12. Cengiz B, Gunduz E, Gunduz M, Beder LB, Tamamura R, Bagci C, Yamanaka N, Shimizu K and Nagatsuka H: Tumor-specific mutation and downregulation of ING5 detected in oral squamous cell carcinoma. Int J Cancer 127: 2088-2094, 2010.

13. Qi L and Zhang Y: Truncation of inhibitor of growth family protein 5 effectively induces senescence, but not apoptosis in human tongue squamous cell carcinoma cell line. Tumour Biol 35: 3139-3144, 2014.

14. Li X, Nishida T, Noguchi A, Zheng Y, Takahashi H, Yang X, Masuda S and Takano Y: Decreased nuclear expression and increased cytoplasmic expression of ING5 may be linked to tumorigenesis and progression in human head and neck squamous cell carcinoma. J Cancer Res Clin Oncol 136: 1573-1583, 2010.

15. Zheng HC, Xia P, Xu XY, Takahashi H and Takano Y: The nuclear to cytoplasmic shift of ING5 protein during colorectal carcinogenesis with their distinct links to pathologic behaviors of carcinomas. Hum Pathol 42: 424-433, 2011.

16. Xing YN, Yang X, Xu XY, Zheng Y, Xu HM, Takano Y and Zheng HC: The altered expression of ING5 protein is involved in gastric carcinogenesis and subsequent progression. Hum Pathol 42: 25-35, 2011.

17. Kumada T, Tsuneyama K, Hatta H, Ishizawa S and Takano Y: Improved 1-h rapid immunostaining method using intermittent microwave irradiation: Practicability based on 5 years application in Toyama Medical and Pharmaceutical University Hospital. Mod Pathol 17: 1141-1149, 2004.

18. https://www.ncbi.nlm.nih.gov/pmc/articles/PMC540017/\# sec22title

19. Shah S, Smith H, Feng X, Rancourt DE and Riabowol K: ING function in apoptosis in diverse model systems. Biochem Cell Bio 87: 117-125, 2009.

20. Gong W, Russell M, Suzuki K and Riabowol K: Subcellular targeting of p33ING1b by phosphorylation-dependent 14-3-3 binding regulates p21WAF1 expression. Mol Cell Biol 26: 2947-2954, 2006

21. Yu L, Thakur S, Leong-Quong RY, Suzuki K, Pang A, Bjorge JD, Riabowol K and Fujita DJ: Src regulates the activity of the ING1 tumor suppressor. PLoS One 8: e60943, 2013.

22. Chen G, Wang Y, Garate M,Zhou J and Li G: The tumor suppressor ING3 is degraded by SCF (Skp2)-mediated ubiquitin-proteasome system. Oncogene 29: 1498-1508, 2010.

This work is licensed under a Creative Commons Attribution 4.0 International (CC BY 4.0) License. 